Cahiers $d u$ MONDE RUSSE

\section{Cahiers du monde russe}

Russie - Empire russe - Union soviétique et États indépendants

$55 / 3-4 \mid 2014$

Varia

\title{
Isaiah Gruber, Orthodox Russia in Crisis, Church and Nation in the Time of Troubles
}

\section{Aleksandr Lavrov}

\section{(2penEdition}

\section{Journals}

Édition électronique

URL : http://journals.openedition.org/monderusse/8018

DOI : 10.4000/monderusse.8018

ISSN : $1777-5388$

\section{Éditeur}

Éditions de l'EHESS

\section{Édition imprimée}

Date de publication : 1 juillet 2014

Pagination : $338-344$

ISBN : 978-2-7132-2441-6

ISSN : $1252-6576$

\section{Référence électronique}

Aleksandr Lavrov, «Isaiah Gruber, Orthodox Russia in Crisis, Church and Nation in the Time of Troubles », Cahiers du monde russe [En ligne], 55/3-4 | 2014, mis en ligne le 08 avril 2015, Consulté le 24 septembre 2020. URL : http://journals.openedition.org/monderusse/8018 ; DOI : https://doi.org/ 10.4000/monderusse.8018

Ce document a été généré automatiquement le 24 septembre 2020.

(c) École des hautes études en sciences sociales 


\title{
Isaiah Gruber, Orthodox Russia in Crisis, Church and Nation in the Time of Troubles
}

\author{
Aleksandr Lavrov
}

\section{RÉFÉRENCE}

Isaiah Gruber, Orthodox Russia in Crisis, Church and Nation in the Time of Troubles, DeKalb : Northern Illinois University Press, 2012, 290 p., ill.

Dans toute étude, une claire définition du sujet est gage de la moitié du succès. Dans le cas d'Isaiah Gruber, on retrouve, dès le titre, au moins trois notions clés : la crise, l'Église et la nation. Une courte mise au point est ici nécessaire. Alors que la notion de crise est devenue une des plus populaires parmi les historiens de la première modernité, sa place dans le titre de cet ouvrage ne me parait pas évidente ${ }^{1}$. En effet, si plusieurs structures fondamentales de la Moscovie semblent avoir éprouvé une crise pendant le Temps des Troubles, ce ne fut pas le cas de l'identité orthodoxe. Pour préciser cet important point de désaccord, on pourrait dresser un bilan : le Temps des Troubles ne provoqua aucun schisme à l'intérieur de l'Église russe orthodoxe ni de conflit entre deux ou plusieurs juridictions, car tous les prétendants au trône du patriarche se réclamaient patriarches de Moscou. Aucune conversion massive des Moscovites à une autre confession n'eut lieu, à l'exception des territoires annexés par la Suède ou par la République lithuano-polonaise, où les conversions s'inscrivirent dans un cadre chronologique très large, allant au-delà du Temps des Troubles. Ainsi, l'identité confessionnelle de l'« orthodox Russia » ne me semble pas mise en question.

Quant à la nation (nation), sa présence en Moscovie au commencement du XVII ${ }^{\mathrm{e}}$ siècle mérite d'être mieux argumentée. Serhii Plokhy, l'auteur de l'étude la plus nuancée de ce phénomène, préfère parler de "protonation ", un terme qui paraît plus approprié dans le cadre étudié ${ }^{2}$. De plus, Isaiah Gruber met sur le même plan patriotisme et 
nationalisme (p. 4), ce qui ne paraît pas évident, car il aurait été tout aussi intéressant de considérer ce " patriotisme » comme un phénomène à part qui se cristallise pendant le Temps des Troubles. ${ }^{3}$

3 Enfin, la notion la plus épineuse, celle de l'Église (Church). L'auteur aurait pu préciser son sujet en choisissant la vision postweberienne, mais il a préféré l'élargir, car en réalité, son étude ne porte pas seulement sur l'Église mais sur la religion en général, y compris toutes les questions de la légitimation religieuse du pouvoir séculier. Si on se souvient que la grande majorité des écrits politiques du Temps des Troubles fut rédigée par des représentants du clergé, ce qui ne signifie pas que ces derniers exposaient dans leurs écrits un point de vue particulièrement clérical, la mission de l'auteur commence à paraître impossible. En réalité, un sujet aussi vaste demande un magnum opus, du type des Guerriers de Dieu de Denis Crouzet. La dernière possibilité de s'en sortir réside dans la chronologie soit ne traiter que la première phase du Temps des Troubles et définir cette publication comme un premier volume. Et de fait, Gruber est proche de cette solution : la majeure partie de sa monographie est consacrée à cette première phase, notamment à l'élection de Boris Godunov et à la famine de 1601-1602, tandis que les activités du patriarche Germogen ne sont qu'esquissées.

Dans son introduction, l'auteur présente le Temps des Troubles dans la mémoire collective russe et dans l'historiographie. Il montre ensuite comment la dénomination "Temps des Troubles» (Smuta) s'est réactualisée, servant à l'interprétation des révolutions de 1917 et de la guerre civile, surtout dans les écrits des émigrés russes. Les pages consacrées à l'historiographie russe de l'Ancien Régime sont marquées d'une clarté exceptionnelle. En revanche, la présentation de l'historiographie soviétique est un peu confuse. Gruber se hâte de déclarer Mihail N. Pokrovskij créateur de la version soviétique du Temps des Troubles, avec notamment sa fameuse "guerre paysanne». Sur cette question, une telle simplification est dangereuse. Tout d'abord, Pokrovskij ne donna pas une, mais plusieurs versions du Temps des Troubles, et même la révolte d'Ivan Bolotnikov qui, selon la version soviétique, formait l'épicentre de cette fameuse "guerre paysanne", fut traitée par lui soit comme un mouvement paysan, soit comme une action de la coalition de nobles et de cosaques ${ }^{4}$. De plus, pour Pokrovskij, ainsi que pour les autres historiens-marxistes de sa génération, la notion de "guerre paysanne " faisait allusion au livre de Friedrich Engels (La guerre des paysans en Allemagne), où la guerre paysanne adopte les traits de la révolution bourgeoise. Cette allusion fut perdue dans l'historiographie soviétique d'après-guerre. Par conséquent, les fidèles continuateurs de Pokrovskij, qui ont eu la chance de survivre à la liquidation de son école, ne s'alignèrent pas sur la «ligne générale ", mais présentèrent une alternative dogmatique à cette dernière, comme en témoigne le cas de Dmitrij P. Makovskij ${ }^{5}$. La «ligne générale» de l'historiographie soviétique fut formée par l'amalgame entre la conception de Pokrovskij (dont on reprit le schéma qui faisait commencer la crise par les émeutes pendant la famine, et non par la crise dynastique), celle de Platonov et celle de Kostomarov, dont on reprend l'image défigurée de l'«intervention" polonaise. Le résultat en fut un phénomène bicéphale, formé par la "guerre paysanne » et l'« intervention étrangère ». En revanche, le rôle d'Aleksandr L. Stanislavskij et de Ruslan G. Skrynnikov dans la rupture avec la notion de "guerre paysanne », remplacée par la " guerre civile », est très bien apprécié par Gruber.

5 Le premier chapitre est consacré à la vision moscovite des relations entre l'État et l'Église. En s'appuyant sur l'article séminal de Daniel Rowland, Gruber affirme que les 
literati moscovites représentaient leur politeia dans des catégories vétérotestamentaires, en la caractérisant comme le « Nouvel Israël ». Pourtant, il est incorrect d'affirmer que " with the possible or partial exception of Ethiopia, no other state in medieval and early modern times appears to have held such a view as its official and exclusive ideology» (p. 26). Comme Marie-Hélène Congurdeau l'a montré, une idéologie identique avait été présente à Byzance ${ }^{6}$.

6 Selon Gruber, le modèle moscovite ne laissait pas de place à des conflits fondamentaux entre l'État et l'Église, car les obligations du tsar étaient aussi perçues comme religieuses. Même le conflit du tsar Ivan Groznyj avec le métropolite Filipp (Kolyčev), qui s'acheva par le meurtre de ce dernier, ne devait pas changer cette donne, car « the Orthodox Church neither desired nor pursued something so antithetial to the goals of its own political ideology»(p.25). La dernière affirmation mérite d'être mieux argumentée. Les études récentes de Vladimir A. Kolobkov et de I.A. Lobakova sur la tradition littéraire de la Vie du métropolite Filipp montrent que les textes hagiographiques séminaux, contenant une critique importante de l'arbitraire du pouvoir séculier à l'égard de l'Église, furent écrits à partir de 1584, c'est-à-dire pendant la période étudiée par l'auteur?.

7 Le deuxième chapitre est consacré aux fondements économiques des grandes communautés monastiques en Russie. Ce chapitre s'appuie sur la recherche précédente de l'auteur et est sans doute le plus original du livre. Gruber caractérise les grandes communautés monastiques moscovites comme des "corporations", avec leur propre conseil d'administration, etc. Selon Gruber, les monastères étaient tournés vers les affaires et le profit («business- and profit oriented», p. 54), et ne différaient pas dans leurs activités économiques des autres acteurs moscovites (p. 59). Ici on peut faire trois remarques. Premièrement, il est préférable de ne parler d'une orientation vers le profit que dans les cas où les monastères investissaient vraiment leurs revenus venant de l'agriculture dans l'achat de propriétés foncières et obtenaient ainsi de nouveaux revenus. Pourtant, le fonctionnement réel qu'on connaît était tout autre - l'extension des domaines des monastères n'était pas due seulement aux achats, mais aussi aux dons. Et que faire des cas où les surplus furent investis soit dans l'entretien d'une plus grande communauté monastique, soit dans des constructions sans but économique immédiat, par exemple, des églises ou des fortifications ? Doit-on simplifier à l'extrême et voir dans ces derniers des investissements dans l'infrastructure? On pourrait supposer au contraire que le mode d'activité économique d'un monastère moscovite s'approchait de l'idéal que cherchent désespérément dans le passé les adeptes actuels $\mathrm{du}$ « développement durable ». Deuxièmement, en partageant l'attention de l'historien sur le fonctionnement économique des monastères moscovites, on est obligé de remarquer que les domaines diocésains (arhierejskie doma) fonctionnaient généralement de la même manière. Troisièmement, une autre particularité des monastères, facilitée par leur prospérité économique, les rendait extrêmement importants dans le Temps des Troubles: leurs fortifications. Il ne faut pas oublier qu'on parle d'un pays où le château aristocratique était un phénomène inconnu. Tandis qu'une ville moscovite, $\mathrm{y}$ compris le siège épiscopal, restait parfois faiblement fortifiée, les murs des monastères, construits aux $\mathrm{XIV}^{\mathrm{e}}-\mathrm{XVI}^{\mathrm{e}}$ siècles en vue de contrer les invasions tatares, permettaient au commencement du XVII ${ }^{\mathrm{e}}$ siècle de tenir tête aux ambitions des divers warlords, dont les armées ravageaient le pays. Pour prouver cette dernière thèse, une comparaison du destin d'une ville assiégée avec celui d'un monastère assiégé est instructive. La ville de 
Smolensk, qui possédait les fortifications les plus modernes de Moscovie, tombe après un siège par l'armée de la Couronne lithuano-polonaise. Mais le monastère de la Trinité-Saint-Serge réussit à tenir tête à une des armées du Second Dmitrij (le « félon de Tušino »).

8 Dans le troisième chapitre de la monographie ("Vox populi, vox Dei et vox feminae ») l'auteur tente de comparer le rôle des trois acteurs principaux dans la légitimation des nouveaux tsars à partir de 1598. Dès l'élection de Boris Godunov (1598) le rôle de l'Église, et celui, personnel, du patriarche Iov, semblent très importants, et ici l'auteur se solidarise avec l'opinion commune de la recherche. Mais pendant la même élection de 1598 une nouvelle figure apparaît: celle de la tsarine douairière. Ainsi, la tsarine Irina, la sœur de Boris Godunov, contribua beaucoup à la légitimation de ce dernier comme tsar. De plus, dans la Décision de l'assemblée (Sobornoe opredelenie) apparaît pour la première fois la formule selon laquelle « la voix du peuple est celle de Dieu ». Gruber montre que cette formule est présente en Occident médiéval dès l'époque de Charlemagne, et qu'elle ne fut incorporée dans le discours moscovite qu'en 1598. Les conclusions de l'auteur sont parfois intéressantes, mais quant à sa source principale - la Décision de l'assemblée de même que la Charte électorale (utverždennaja gramota) de l'assemblée des états de 1598 - il existe une étude de l'histoire de ce texte, écrite par Svetlana Mordovina, qui n'est pas même citée ${ }^{8}$. C'est pourquoi la comparaison des deux points de vue sur l'Assemblée, - sceptique par Sergej F. Platonov, et positive par Chester Dunning - proposée par Gruber, n'est pas convaincante.

9 Le quatrième chapitre («Famine et douleur») porte sur la réponse de l'Église à la famine et à l'aventure du Premier Dmitrij. Gruber montre que les monastères disposaient de grandes ressources en grain, y compris pendant les années de la famine. Son analyse des revenus de la procure du monastère de Solovki à Vologda montre que pendant les années de famine le monastère a vendu plus de grain, profitant de la multiplication des prix par deux. L'auteur conclut avec raison qu'une telle pratique pouvait entraîner «chez une population affamée une perception négative des monastères et des ecclésiastiques » (p. 103).

10 Le cinquième chapitre ("La Maison divisée ») étudie le rôle de l'Église au temps de Vasilij (Šujskij) et du Second Dmitrij, quand le «vrai » tsar, résidant à Moscou, et le prétendant, résidant à Tušino, avaient chacun leur patriarche. L'auteur remarque à juste titre que la légitimation du tsar Vasilij s'inscrit largement dans un modèle connu : il se prétend " élu » par une Assemblée des états, le clergé le prie de monter sur le trône, et la mère du vrai Dmitrij confirme que son fils est mort (vox feminae) (p.129-130,133-134). Cependant, l'auteur reconnait lui-même que ces arguments n'étaient efficaces qu'à Moscou et ses environs (p.137), ce qui amène à se demander dans quelle mesure la question de la légitimation était cruciale pour cette phase du Temps des Troubles. En même temps, la légitimation du « Second Dimitrij » semblait se construire selon le même scénario - même si la vox populi manqua à Tušino, la vox feminae était en jeu - et ce dernier témoignage était unique. Bien que l'auteur penche plutôt vers une opposition entre deux légitimations, quand il parle ici de l'Orthodoxy of rebellion (p.139), il me semble que le tsar Vasilij et le "Second Dmitrij" faisaient généralement la même chose. En dehors de la partie consacrée à la dynamique des revenus des monastères pendant ce temps, ce chapitre est le moins original et étudie surtout les événements à Moscou ou dans ses environs. On peut déplorer que l'auteur n'ait pas ajouté un cadre local (par exemple, celui d'un diocèse). 
11 Le sixième et dernier chapitre («Mort et résurrection. La culmination et la fin du Temps des Troubles ») est consacré au rôle de l'Église dans l'organisation des deux Coalitions (opolčenie). Selon Gruber, l'idéologie du Nouvel Israël donnait des possibilités pour une interprétation affirmative aussi bien que subversive. Les guerres et les troubles pouvaient être interprétés comme un châtiment divin pour la non-obéissance aux commandements de Dieu, contribuant ainsi au soutien de la critique des autorités. Cette dernière interprétation subversive contribua largement à la formation de la conscience nationale russe, nommée par l'auteur «orthodoxie populaire » (popular Orthodoxy), à laquelle se référaient les «forms of unofficial and unauthorized belief opposed to the official pronouncements of church and state » (p. 153).

12 Cette vision est très intéressante, mais nous devons formuler certaines critiques. Premièrement, la notion d'« orthodoxie populaire » n'est pas nouvelle, elle fut utilisée par plusieurs chercheurs comme un homologue de la « religion populaire " à la russe (ou à l'ukrainienne). Étant donné que les écrits cités par l'auteur furent composés par des représentants des élites ecclésiastiques moscovites, la notion choisie ne semble pas appropriée. Deuxièmement, en postulant un rôle formateur du message ecclésiastique pour la conscience nationale, on est obligé de préciser les moyens de communication par lesquels ce message était transmis aux fidèles. Le sous-développement de la culture de l'imprimé, l'absence de la prédication et l'analphabétisme de la grande partie de la population constituent les obstacles principaux à cette hypothèse.

13 Le défaut principal de la présentation de l'auteur vient de sa structure chronologique. Il aurait été de loin préférable de choisir une structure thématique qui étudiât la situation de plusieurs groupes du clergé, y compris les évêques, le clergé régulier et séculier. Cette structure aurait permis à l'auteur de mieux mettre ses trouvailles en valeur, alors que du fait de cette structure chronologique, elles se perdent dans la présentation de faits déjà connus.

14 Elle aurait permis de présenter le rôle non seulement des patriarches, mais aussi des évêques. La composition personnelle du corps des évêques pendant le Temps des Troubles n'est pas même effleurée par l'auteur bien que l'intérêt pour cette question soit légitime : les changements personnels dans la Douma de boyards pendant le Temps des Troubles sont étudiés depuis des décennies et servent de repères importants dans les méandres de la politique moscovite ${ }^{9}$. L'auteur observe une fois les sympathies d'un représentant du clergé séculier à l'égard des "faux tsars ", mais ce cas n'est pas replacé dans son contexte (p. 138) ${ }^{10}$. Seule la situation des grandes corporations monastiques pendant le Temps des Troubles est systématiquement étudiée dans la monographie.

15 En analysant les événements assez connus du Temps des Troubles l'auteur s'appuie parfois sur la littérature secondaire sans un recours pourtant obligatoire aux sources, ce qui le conduit parfois à des imprécisions. Ainsi, en citant le métropolite Makarij (Bulgakov) et Chester Dunning, l'auteur affirme que la Douma de boyards (the boyard council) «annonça la déposition d'Ignatii » (p.129). L'annonce de la déposition du patriarche par la Douma de boyards semble bien étrange du point de vue canonique. Puisqu'aucun cas semblable n'est connu, on pourrait parler dans le cas d'Ignatij d'une exception unique. Mais le métropolite Makarij (Bulgakov), cité par l'auteur, parle clairement d'une décision du concile ecclésiastique, qui n'était pas difficile à convoquer grâce à la présence élargie des évêques dans la capitale du fait du mariage du Premier Dmitrij ${ }^{11}$. On pourrait faire la même critique de l'affirmation de l'auteur selon lequel « the capital city of Moscow » fut libérée par la Deuxième Coalition le 4 novembre 1612. 
En réalité, seul Kitaj-gorod fut libéré, tandis que le Kremlin restait sous contrôle de la garnison polonaise. La capitulation fut signée le jour suivant et la garnison polonaise rendit les armes seulement le 6 novembre ${ }^{12}$. Enfin certaines traductions pourraient être critiquées. Ainsi antimins est traduit comme "holy communion cloth» (p.157). En réalité, l'antimins (ou antimension, comme on le translittère normalement en anglais) était « a cloth of linen or silk to which relics were attached and on which scenes of the Passion, usually Christ's entombment, were depicted», qui " originally served as a portable altar $»^{13}$.

De façon générale, l'ouvrage porte l'empreinte d'un grand effort, entrepris par l'auteur; l'inventaire de sources et de la littérature secondaire qu'il a utilisé est impressionnant. Il reste à regretter que l'auteur n'ait pas osé choisir un titre plus précis et mieux ciblé, qui eût mieux correspondu au travail accompli.

\section{NOTES}

1. Voir l'ouvrage de synthèse: Geoffrey Parker, Global Crisis, War, Climate Change and Catastrophe in the Seventeenth Century, New Haven :Yale University Press, 2013.

2. Serhii Plokhy, The origins of the Slavic nations: Premodern identities in Russia, Ukraine, and Belarus, Cambridge, 2006. Voir notre compte-rendu: Annales. Histoire, Sciences Sociales, 64 (3), 2009, p. 709-711.

3. M.M. Krom "Skol'ko let patriotizmu? [Quel âge a le patriotisme]», Rodina, n 10, 1994, p. 15-21.

4. Voir une critique très remarquable des diverses visions proposées par Pokrovskij à partir de la version soviétique officielle dans l'article d'A. Savič. Savič est surtout indigné de l'inconséquence de Pokrovskij à propos du Premier Dmitrij, qu'il présente soit comme un «tsar des nobles (dvorjanskij car') », soit comme « un tsar anti-noble (protivodvorjanskij car') ». A. Savič, « Pol'skaja intervencija načala XVII v. v ocenke M.N. Pokrovskogo [L'intervention polonaise du début du $\mathrm{XVII}^{\mathrm{e}}$ siècle, vue par M.N. Pokrovskik]», Protiv antiistoričeskoj koncepcii M.N. Pokrovskogo : Sbornik statej [Contre la conception anti-historique de M.N. Pokrovskij, recueil d'articles] M.-L., 1939, 2, p. 179-243.

5. D.P. Makovskij, Pervaja krest'janskaja vojna $v$ Rossii [La première guerre paysanne en Russie], Smolensk : Moskovskij rabocij, 1967.

6. Marie-Hélène Congurdeau, «Le judaïsme, cœur de l'identité byzantine », in M. Dmitriev, D. Tollet et E. Teiro, éds., Les Chrétiens et les Juifs dans les sociétés de rites grec et latin. Approche comparative, P. : Honoré Champion, 2003, p. 17-27.

7. V.A. Kolobkov Mitropolit Filipp i stanovlenie moskovskogo samoderžavija: Opričnina Ivana Groznogo [Le métropolite Filipp et l'établissement de l'autocratie moscovite : l'Opričnina d'Ivan le terrible], SPb., 2004 ; I.A. Lobakova Žitie mitropolita Filippa: issledovanie i teksty [Biographie du métropolite Filipp : recherches, textes] SPb., 2006 ;

8. S.P. Mordovina, « K istorii utverždennoj gramoty 1598 g. [L'histoire de la Charte électorale de 1598]», Arheografičeskij ežegodnik za 1968 god, M., 1970.

9. On pourrait dire que le texte, tel qu'il est publié, présente une histoire politique du patriarcat de Moscou pendant le Temps des Troubles. C'est notamment dans cette perspective qu'on peut regretter trois lacunes dans la bibliographie: la monographie ecclésiastique préparée pour la 
canonisation du patriarche Germogen, la thèse consacrée aux activités de Filaret (Romanov) pendant le Temps des Troubles et la synthèse la plus récente de l'histoire du patriarcat de Moscou : S. Kedrov Žizneopisanie svjatejšego Germogena, patriarha moskovskogo i vseja Rossii [La vie de Germogen, patriarche de Moscou et de toute la Russie], M., 1912 ; Gisela Brigitte Bernhard, Feodor Nikitič Romanov / Filaret : seine Politik in der Zeit der Smuta (1598-1613), Heidelberg, 1977 ; Wolfram von Scheliha, Russland und die orthodoxe Universalkirche in der Patriarchatsperiode (1589-1721), Wiesbaden, 2004.

10. On pourrait trouver des cas identiques dans le clergé régulier, par exemple, chez le «černyj pop » Anton du monastère Modenskij Nikolaevskij, qui a lu publiquement une lettre du Premier Dmitrij (N.P. Voskobojnikova, Opisanie drevnejših dokumentov arhivov moskovskih prikazov XVI-nač. XVII vv. (RGADA, f.141. Prikaznye dela staryh let) [Description de documents anciens des archives des prikazy moscovites XVI ${ }^{\mathrm{e}}$-début du XVII ${ }^{\mathrm{e}}$ (RGADA, f. 141)], M., 1994, p. 222.

11. Makarij (Bulgakov), Istorija russkoj Cerkvi [Histoire de l'Église russe], t. 10, Patriaršestvo v Rossii [Le patriarcat en Russie], kn. 1, SPb., 1881, p. 122.

12. Vladislav Nazarov, « Čto budut prazdnovat' v Rossii 4 nojabrja 2005 goda [Que fêterons-nous en Russie le 4 novembre 2005 ?]» // Polit.ru/article/2005/04/22/prazdniki/, dernière consultation le 20 octobre 2013.

13. James Cracraft, The Petrine Revolution in Russian Imagery, Chicago - London, 1997, p. 150-151.

\section{AUTEURS}

\section{ALEKSANDR LAVROV}

Université Paris IV - Sorbonne 